\title{
Comparison between FIB-4 Index and Fibroscan as Marker of Fibrosis in Chronic HCV Infection in Egyptian Patients
}

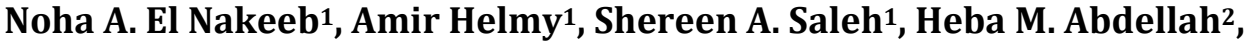 \\ Mostafa H. Abdel Aleem², Dina Elshennawy ${ }^{3}$ \\ ${ }^{1}$ Internal Medicine Department, Faculty of Medicine, Ain Shams University, Cairo, Egypt \\ ${ }^{2}$ Tropical Medicine Department, Faculty of Medicine, Ain Shams University, Cairo, Egypt \\ ${ }^{3}$ Clinical Pathology Department, Faculty of Medicine, Ain Shams University, Cairo, Egypt \\ Email: hbabdella@yahoo.com
}

Received 5 October 2014; revised 23 November 2014; accepted 6 December 2014

Copyright (C) 2014 by authors and Scientific Research Publishing Inc.

This work is licensed under the Creative Commons Attribution International License (CC BY). http://creativecommons.org/licenses/by/4.0/

c) (i) Open Access

\section{Abstract}

The FIB-4 index is a simple and noninvasive algorithm consisting to evaluate liver fibrosis in chronic HCV infection. Aim: To evaluate the utility of FIB-4 index as a noninvasive marker to assess liver fibrosis in chronic HCV infection in comparison to transient elastography. Patients and Methods: We studied 30 patients having chronic HCV infection based on clinical features, laboratory tests, diagnostics images, Fibroscan and FIB-4 score. According to the results of Fibroscan, the 30 patients were classified into two groups in order to obtain a cutoff value to exclude patient with significant fibrosis: group Ia: 7 patients with no or mild liver fibrosis (F0-F1) and group Ib: 23 patients with significant fibrosis or cirrhosis (F2-F3-F4). Group IIa: 17 patients with no or significant fibrosis (F0-F1-F2-F3) and group IIb (F4): 13 patients with cirrhosis (F4). Results: The mean of FIB-4 index increased with the increase of the fibrosis score. FIB-4 index proved to be sensitive and specific in differentiation between patients with no or mild fibrosis (F0-F1) and patients with significant fibrosis or cirrhosis (F2-F3-F4) with the best cutoff value at 1.61. It also proved to be sensitive and specific in differentiation between patients with no or significant fibrosis (F0-F1F2-F3) and patients with cirrhosis (F4) with cutoff value at 1.88. Conclusion: The FIB-4 index enabled the correct identification of extreme types of fibrosis. Using these cutoffs (1.61 - 1.88), 87\% of patients fell outside these ranges and could thus avoid liverbiopsy with an overall accuracy of 70\%.

\section{Keywords}

Chronic HCV Infection, Transient Elastography, FIB-4, Liver Fibrosis, Liver Cirrhosis 


\section{Introduction}

Hepatitis C virus (HCV) is a leading cause of liver cirrhosis and cancer. Egypt has possibly the highest HCV prevalence worldwide [1]. The prognosis and management of a patient with chronic hepatitis $\mathrm{C}$ virus (HCV) infection depend very much on the severity of liver fibrosis [2]. At present, the golden standard to assess liver fibrosis is a liver biopsy using the Ishak [3] or Metavir scores [4]. However, biopsy is prone to sampling error and substantial intra and inter observer variability, leading to over or under staging of fibrosis [5]. Needle biopsy of the liver is also clearly unsuitable for monitoring disease progression, especially in untreated patients. For these reasons, non-invasive methods for assessing the severity of fibrosis may someday completely replace liver biopsy and are constantly being searched for. Among the non-invasive tests, the best results were obtained with liver stiffness measurement (LSM) by means of transient elastography (TE) (Fibroscan). This non-invasive method is expensive and requires equipment that is not widely available [6]. Therefore simpler, cheaper methods for the prediction of hepatic fibrosis were sought for. As a fact, Fibroscan is more and more used in routine clinical practice as an alternative method to liver biopsy in patients with chronic liver diseases [7]. Main physical limitation is linked to the presence of ascites as liquids prevent the propagation of shear waves. As a consequence, shear waves are stopped before they can enter liver, obesity and more specifically to a large skin to liver capsula distance. Severe flares of hepatitis (ALT > $10 \times \mathrm{ULN}$ ), during which the liver stiffness value may be spuriously high, returning to normal levels after resolution of the flares. Therefore, transient elastography performed at the time of severe flares will lead to over-diagnosis of severe fibrosis and cirrhosis. Fibroscan is contraindicated on any other organ than the liver or on patient with active implantable devices such as pacemakers, defibrillators, pumps, etc., on wounds or in pregnancy or in ascites [8]. The investigators of the AIDS Pegasys Ribavirin International Coinfection Trial (Apricot study) [9] proposed a simple noninvasive test for liver fibrosis known as the FIB-4-a test derived from the Apricot database, which produces interesting results using the following formula: age $($ years $) \times \operatorname{AST}[\mathrm{U} / \mathrm{L}] /\left(\right.$ platelet $\left.\left[10^{9} / \mathrm{L}\right] \times(\mathrm{ALT}[\mathrm{U} / \mathrm{L}]) 1 / 2\right) \quad[10]$. Platelet count is known to correlate with the amount of portal hypertension and advanced fibrosis. Elevations in AST more than ALT have also been associated with more advanced fibrosis and are in part related to delayed clearance of AST relative to ALT or to mitochondrial injury associated with more advanced fibrosis [11]. The FIB-4 index performed at least as well or better than a more complicated model using tests not readily available in routine clinical practice [12].

Aim of the work was to evaluate the utility of FIB-4 index as a simple inexpensive non invasive marker to assess liver fibrosis in Egyptian patients with chronic HCV infection in comparison to transient elastography (TE) (Fibroscan).

\section{Patients and Methods}

This study was conducted on patients attending outpatient clinics at the departments of internal and Tropical Medicine at Ain Shams University from: June 2013 to March 2014. All patients were subjected to the following:

Full medical history with special stress on: Previous history of chronic liver disease, history of viral hepatitis or exposure to risk factors (such as tarter emetic injections, blood transfusion, operations... etc.), upper and lower gastrointestinal bleeding, symptoms of liver cell failure such as jaundice, lower limb edema, abdominal enlargement, bleeding tendency... etc., recent use of hepatotoxic drugs or Alcohol consumption and previous history of immune thrombocytopenic purpra (ITP). Clinical examination was done with special stress on liver and splenic size, ascites and signs of liver cell failure as jaundice, palmer erythema, lower limb edema or encephalopathy... etc. Laboratory investigations included complete blood picture including platelets count, coagulation profile: prothrombin time (PT), and international normalized ratio (INR) by standard lab tests, liver profile: Aspartate aminotransferase (AST), alanine aminotransferase (ALT), total and direct serum bilirubin, and serum albumin, by standard laboratory tests, renal profile: Serum creatinine and Blood urea nitrogen (BUN), serum Alpha fetoprotein, lipid profile, autoimmune markers: Anti nuclear antibodies (ANA) and Anti smooth muscle antibodies (ASMA) and viral markers for HCV and HBV: HBsAg, HBsAb, HBcAb IgM, and HCV Antibodies by ELISA. Abdominal ultrasonography was done to all patients.

Patients underwent transient elastography (Fibroscan) examination:

- Equipment: Fibroscan, 502.

- The patient lies on his back with his arm raised behind his head. The physician applies water based gel to the skin and places the probe with a slight pressure. The examination included 10 consecutive measurements made to the same location. The result delivered at the end of the examination, in a form of a number which 
can vary from 1.5 to $75 \mathrm{Kpa}$. The cutoff values of the Fibroscan in hepatitis C virus monoinfection were measured according to Ziol et al., 2005 [6].

- F0-F1: 0 - 7 Kpa

- F1-F2: 7 - $8.5 \mathrm{Kpa}$

- F2: 8.5 - $9.5 \mathrm{Kpa}$

- F3: $9.5-12.5 \mathrm{Kpa}$

- F3-F4: 12.5 - $14.5 \mathrm{Kpa}$

- F4: 14.5 - $75 \mathrm{Kpa}$

Exclusion criteria: Chronic hepatitis B infection, chronic alcohol abuse, fatty liver, autoimmune hepatitis, patients with hepatic focal lesion by abdominal ultrasound, patient with ITP, patient receiving hepatotoxic drugs with elevated liver enzymes and liver cell failure.

Then, 30 patients with confirmed diagnosis of chronic HCV infection and liver fibrosis were selected. The diagnosis was based on clinical features, laboratory test, diagnostics images, and transient elastography.

The selected patients were subjected to calculation of the FIB-4 score as follows [10].

The FIB-4 score was calculated according to the formula:

$$
[\text { age }(\text { years }) \times \operatorname{AST}(\mathrm{IU} / \mathrm{L})] /\left[\text { platelet count }\left(10^{9} / \mathrm{L}\right) \times \operatorname{ALT}(\mathrm{IU} / \mathrm{L}) 1 / 2\right]
$$

For easier statistics and comparisons to have valid results and valid cutoff values, patients were classified by Fibroscan into 2 groups by 2 different ways:

1) To exclude patients with significant fibrosis

- Group Ia: 7 patients with no or mild liver fibrosis (F0-F1).

- Group Ib: 23 patients with significant fibrosis or cirrhosis (F2-F3-F4). The patients.

2) Then, they were reclassified in order to obtain a cutoff value to identify patients with cirrhosis from different grades of fibrosis.

- Group IIa: 17 patients with no or significant fibrosis (F0-F1-F2-F3).

- Group IIb: 13 patients with cirrhosis (F4).

\section{Statistical Methods}

Analysis of the data was done by IBM computer using SPSS (statistical package for social science version 16). Data were described as mean \pm standard deviation (SD) for quantitative variables student. Unpaired student t-test was used for comparison of quantitative variable among more than two independent groups. Spearman correlation coefficient ( $r$ ) test was used to rank different variables against each other either positive or inverse. The probability value $(\mathrm{P})<0.05$ and $<0.01$ was considered statistically significant and highly significant respectively, however P-value $>0.05$ was not significant. ANOVA test was used for analysis of variation. Chi-square $\left(\chi^{2}\right)$ test was used to study the association between each 2 variables or comparison between 2 independent groups as regard the categorized data. The receiver operator characteristic (ROC) Curve was constructed to obtain the most sensitive and specific cutoff for each technique. To evaluate the most discriminating markers between the compared groups, AUC can also be calculated. Sensitivity is the proportion of actual positive which are correctly identified. Specificity is the proportion of negative which are correctly identified. NPV (negative predictive value) is the proportion of subjects with a negative test results who are correctly diagnosed. PPV (positive predictive value) is which is the proportion of positive test results that are true positive.

\section{Results}

This study was conducted on 30 patients 24 were males (80\%) and 6 were females (20\%) with their ages ranged from 25 to 60 years with mean \pm SD $49.3 \pm 8.2$ years. According to the results of Fibroscan, 2 patients (6.7\%) were F0 (no fibrosis), 5 patients (16.7\%) were F1 (moderate fibrosis), 5 patients (16.7\%) were F2 (intermediate fibrosis), 5 patients (16.7\%) were F3 (extensive fibrosis), and 13 patients (43.3\%) were F4 (cirrhosis). Table 1 shows the comparison between FIB-4 values in different fibrosis stages measured by Fibroscan. There was a highly significant statistical difference when F1 is compared with F4, when F2 is compared with F4 and when F3 was compared with F4. There was a high statistically significant positive correlation as regard the AST, the results of Fibroscan in K. pascal, total \& direct bilirubin while there was a high statistically significant negative correlation as regard platelet count and serum albumin. There was no statistically significant correlation as re- 
gard the gender, ALT, serum creatinine and Alpha fetoprotein (Table 2). On comparing group Ia (F0-F1) and group Ib (F2-F3-F4), there was highly statistically significant difference as regards results of Fibroscan in K. pascal and there was a statistically significant difference as regard FIB-4 values, platelet count, AST, total bilirubin, direct bilirubin, serum creatinine and white blood count value. On the other hand, the ALT, serum albumin, prothrombin time, international normalization ratio, alpha fetoprotein, and haemoglobin have no statistically significant difference between both groups (Table 3). Receiver operator curve (ROC) of the FIB-4 index for the diagnosis of non significant fibrosis showed a cut-off point of $<1.61$, AUC 91.6, sensitivity $69.57 \%$, specificity 100\%, PPV 100\% and NPV 50\% (Figure 1(a), Figure 1(b)).

Table 1. Comparison between FIB-4 values in different stages of fibrosis measured by Fibroscan.

\begin{tabular}{|c|c|c|c|}
\hline \multirow{2}{*}{ Fibroscan } & \multicolumn{3}{|c|}{ FIB-4 } \\
\hline & Mean \pm SD & \multicolumn{2}{|c|}{ Range } \\
\hline F0 & $0.76 \pm 0.30$ & \multicolumn{2}{|c|}{$0.55-0.97$} \\
\hline $\mathrm{F} 1$ & $0.96 \pm 0.40$ & \multicolumn{2}{|c|}{$0.62-1.61$} \\
\hline F2 & $1.47 \pm 0.80$ & \multicolumn{2}{|c|}{$0.89-2.82$} \\
\hline F3 & $1.75 \pm 0.65$ & \multicolumn{2}{|c|}{$0.93-2.71$} \\
\hline F4 & $3.16 \pm 1.65$ & \multicolumn{2}{|c|}{$1.02-8.01$} \\
\hline \multirow[t]{2}{*}{ ANOVA test } & $F=4.504$ & \multicolumn{2}{|c|}{ P-value $=0.007$} \\
\hline & Groups & P-value & Significance \\
\hline \multirow{8}{*}{ LSD test } & F0 vs F1 & 0.846 & NS \\
\hline & F0 vs F2 & 0.493 & NS \\
\hline & F1 vs F2 & 0.515 & NS \\
\hline & F1 vs F3 & 0.320 & NS \\
\hline & F1 vs F4 & 0.002 & S \\
\hline & F2 vs F3 & 0.724 & NS \\
\hline & F2 vs F4 & 0.015 & S \\
\hline & F3 vs F4 & 0.039 & S \\
\hline
\end{tabular}

NS: Non significant statistical difference; S: Significant statistical difference.

Table 2. Correlation between FIB-4 values and different parameters of the patients in the study.

\begin{tabular}{|c|c|c|c|}
\hline & \multicolumn{2}{|c|}{ FIB-4 } & \multirow{2}{*}{ Significance } \\
\hline & $\mathrm{r}$ & $\mathrm{P}$ & \\
\hline Age (years) & 0.264 & 0.158 & NS \\
\hline AST (U/L) & $0.519^{* * *}$ & 0.003 & S \\
\hline ALT (U/L) & 0.128 & 0.501 & NS \\
\hline $\operatorname{PLT}\left(\times 10^{3} / \mathrm{cmm}\right)$ & $-0.741^{* *}$ & 0.000 & S \\
\hline K. pascal & $0.499^{* *}$ & 0.005 & S \\
\hline Tbil (mg/dl) & $0.503^{* *}$ & 0.005 & S \\
\hline Dbil (mg/dl) & $0.461^{*}$ & 0.010 & S \\
\hline Alb (g/dl) & $-0.431^{*}$ & 0.017 & S \\
\hline Creat (mg/dl) & 0.045 & 0.815 & NS \\
\hline AFP (ng/ml) & 0.329 & 0.076 & NS \\
\hline
\end{tabular}


Table 3. Comparison between group Ia (F0-F1) and group Ib (F2-F3-F4) regarding different parameters, Fibroscan results and FIB-4 value.

\begin{tabular}{|c|c|c|c|c|c|c|c|}
\hline & \multicolumn{2}{|c|}{ Group Ia (F0-F1) } & \multicolumn{2}{|c|}{ Group Ib (F2-F3-F4) } & \multicolumn{2}{|c|}{ Independent t-test } & \multirow{2}{*}{ Significance } \\
\hline & Mean & $\pm \mathrm{SD}$ & Mean & $\pm \mathrm{SD}$ & $\mathrm{T}$ & P-value & \\
\hline AST (U/L) & 30.86 & \pm 20.03 & 64.43 & \pm 33.22 & -2.520 & 0.018 & $\mathrm{~S}$ \\
\hline ALT (U/L) & 44.71 & \pm 30.85 & 81.78 & \pm 67.67 & -1.393 & 0.175 & NS \\
\hline $\operatorname{PLT}\left(\times 10^{3} / \mathrm{cmm}\right)$ & 235.14 & \pm 49.64 & 171.26 & \pm 54.50 & 2.767 & 0.010 & $\mathrm{~S}$ \\
\hline Tbil (mg/dl) & 0.65 & \pm 0.26 & 1.03 & \pm 0.34 & -2.659 & 0.013 & $\mathrm{~S}$ \\
\hline Dbil (mg/dl) & 0.29 & \pm 0.10 & 0.44 & \pm 0.22 & -1.782 & 0.086 & $\mathrm{~S}$ \\
\hline Alb (g/dl) & 4.40 & \pm 0.45 & 4.10 & \pm 0.42 & 1.622 & 0.116 & NS \\
\hline PT (secs) & 13.20 & \pm 0.00 & 13.88 & \pm 1.06 & -0.859 & 0.415 & NS \\
\hline INR & 1.00 & \pm 0.00 & 1.15 & \pm 0.19 & -1.070 & 0.316 & NS \\
\hline Creat (mg/dl) & 0.70 & \pm 0.11 & 0.86 & \pm 0.16 & -2.390 & 0.024 & $\mathrm{~S}$ \\
\hline AFP (ng/ml) & 4.56 & \pm 1.61 & 9.28 & \pm 7.33 & -1.672 & 0.106 & NS \\
\hline WBC (cmm) & 7814.29 & \pm 2015.30 & 6301.74 & \pm 1926.58 & 1.801 & 0.083 & $\mathrm{~S}$ \\
\hline $\mathrm{HB}$ (g/dl) & 14.84 & \pm 1.13 & 15.04 & \pm 1.69 & -0.287 & 0.777 & NS \\
\hline K. pascal (Kpa) & 5.21 & \pm 1.07 & 21.34 & \pm 15.05 & -2.797 & 0.009 & HS \\
\hline FIB-4 & 0.90 & \pm 0.36 & 2.49 & \pm 1.52 & -2.701 & 0.012 & $\mathrm{~S}$ \\
\hline
\end{tabular}

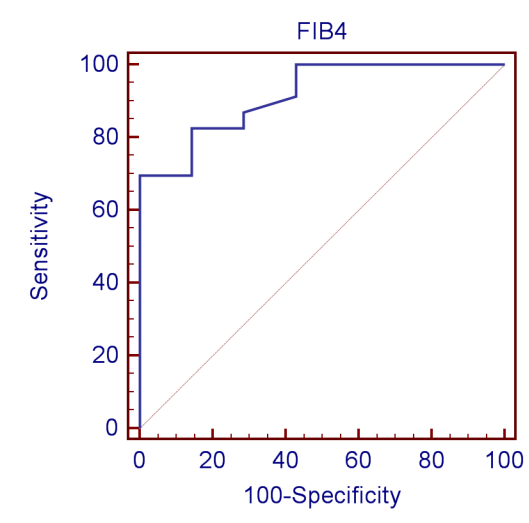

(a)

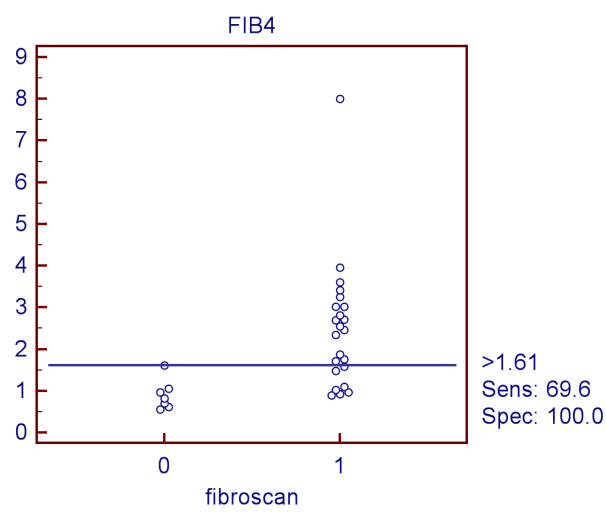

(b)

Figure 1. Receiver operator curve (ROC) of the FIB-4 index for the diagnosis of non significant fibrosis.

On comparing group IIa (F0-F1-F2-F3) and group IIb (F4), there was a high statistically significant difference as regard the Fibroscan results [13] in K. pascal and there was a statistically significant difference as regard the FIB-4 value, AST, direct bilirubin, international normalization ratio, alpha fetoprotein and white blood count platelet count, total bilirubin and serum albumin. Whereas there was no statistically significant difference as regard the ALT, prothrombin time, serum creatinine and haemoglobin (Table 4). Receiver operator curve (ROC) of the FIB-4 index for the diagnosis of cirrhosis showed cut-off point $>1.88$, AUC 90.5, sensitivity 84.6\%, specificity $88.2 \%$, PPV 88.24\% and NPV 100\% (Figure 2(a), Figure 2(b)).

\section{Discussion}

Chronic hepatitis C virus (HCV) infection is emerging as an increasing burden to health and an important cause of morbidity and mortality worldwide [14] [15] with an estimated global prevalence ranging between $2 \%$ to $3 \%$, representing up to 170 million of chronic carriers [16]. Egypt has the highest worldwide prevalence, with 9\% countrywide and up to $50 \%$ in certain rural areas [17]. Hepatitis C virus (HCV) is also the leading cause of liver cirrhosis and cancer in Egypt [1]. 


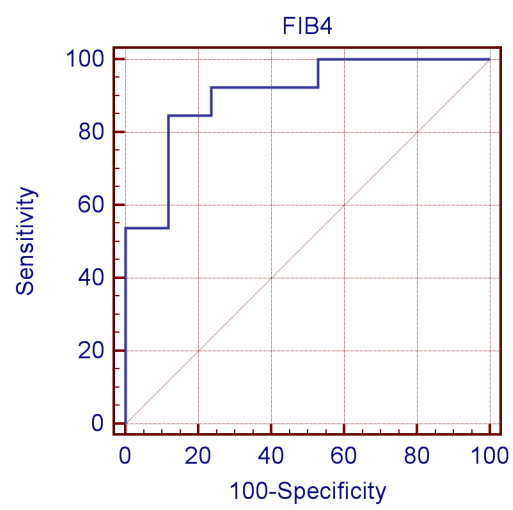

(a)

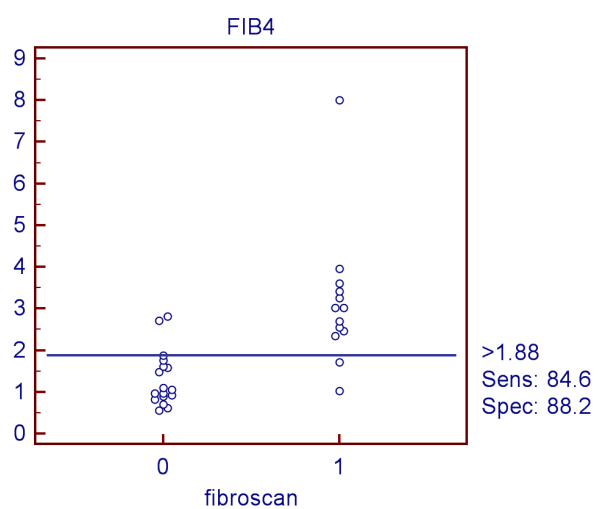

(b)

Figure 2. Receiver operator curve (ROC) of the FIB-4 index for diagnosis of cirrhosis.

Table 4. Comparison between group IIa (F0-F1-F2-F3) and group IIb (F4) as regards different parameter, Fibroscan results and FIB-4 value.

\begin{tabular}{|c|c|c|c|c|c|c|c|}
\hline & \multicolumn{2}{|c|}{ Group IIa } & \multicolumn{2}{|c|}{ Group IIb } & \multicolumn{2}{|c|}{ Independent t-test } & \multirow{2}{*}{ Significance } \\
\hline & Mean & $\pm \mathrm{SD}$ & Mean & $\pm \mathrm{SD}$ & $\mathrm{T}$ & P-value & \\
\hline AST (U/L) & 44.59 & \pm 25.17 & 72.31 & \pm 37.57 & 2.419 & 0.022 & S \\
\hline ALT (U/L) & 61.82 & \pm 37.98 & 87.92 & \pm 84.53 & 1.136 & 0.265 & NS \\
\hline PLT (cmm) & 218.00 & \pm 49.20 & 144.54 & \pm 44.29 & 4.228 & 0.000 & S \\
\hline Tbil (mg/dl) & 0.76 & \pm 0.28 & 1.17 & \pm 0.33 & -3.737 & 0.001 & S \\
\hline Dbil (mg/dl) & 0.32 & \pm 0.14 & 0.51 & \pm 0.23 & -2.749 & 0.01 & S \\
\hline Alb (g/dl) & 4.39 & \pm 0.40 & 3.89 & \pm 0.31 & 3.733 & 0.001 & $\mathrm{~S}$ \\
\hline PT (secs) & 13.20 & \pm 0.00 & 14.28 & \pm 1.19 & -2.022 & 0.078 & NS \\
\hline INR & 1.00 & \pm 0.00 & 1.24 & \pm 0.19 & -2.832 & 0.022 & $\mathrm{~S}$ \\
\hline Creat (mg/dl) & 0.78 & \pm 0.14 & 0.86 & \pm 0.19 & -1.328 & 0.195 & NS \\
\hline AFP (ng/ml) & 5.82 & \pm 2.60 & 11.27 & \pm 9.08 & -2.363 & 0.025 & S \\
\hline WBC $(\mathrm{cmm})$ & 7390.59 & \pm 1973.07 & 5692.31 & \pm 1702.19 & 2.476 & 0.02 & S \\
\hline HB (g/dl) & 15.16 & \pm 1.36 & 14.78 & \pm 1.83 & 0.657 & 0.516 & NS \\
\hline K. pascal (Kpa) & 7.92 & \pm 2.67 & 30.20 & \pm 14.76 & 6.126 & 0.000 & HS \\
\hline FIB-4 & 1.32 & \pm 0.67 & 3.16 & \pm 1.66 & -4.169 & 0.000 & $\mathrm{~S}$ \\
\hline
\end{tabular}

HCV infection is associated with varying degrees of liver inflammation and progressive fibrosis, which may result in cirrhosis and hepatocellular carcinoma [18]. Noninvasive methods estimating HCV-related liver fibrosis were proposed over the last few years. Among those tests, the most important are the AST/ALT ratio, the AST to platelet ratio index (APRI) and the FIB-4 index [10] [19]. The FIB-4 index is a noninvasive method for the evaluation of liver fibrosis, based on simple variables such as age, AST, ALT and platelet count. It was initially proposed by researchers of the APRICOT study (AIDS Pegasys Ribavirin International Coinfection Trial) to evaluate the presence of liver fibrosis in HIV/HCV coinfected patients [10].

In this study on the whole sample, FIB-4 values ranged from 0.55 to 8.01, mean values increased as a function of the fibrosis score, from $0.76 \pm 0.30$ in METAVIR F0 cases to $3.16 \pm 1.65$ in F4. However, the differences were only statistically significant $(\mathrm{P}<0.05)$ when groups with cirrhosis $(\mathrm{F} 4)$ were compared with groups with moderate fibrosis (F1) and intermediate fibrosis (F2). The FIB-4 index could not significantly discriminate F0 from F1 groups, nor F2 from F3. This was in agreement with Vallet-Pichard et al. (2007) [11] who had the same 
results.

There was no statistically significant difference when group Ia (F0-F1) was compared with group Ib (F2-F3F4) and group IIa (F0-F1-F2-F3) with group IIb (F4) as regard the age. This contradict with Sterling et al., 2006 [10] who found that there was statistically significant difference between mild (Ishak score 0 - 1), moderate (Ishak score 2 - 3) and severe fibrosis (Ishak score 4 - 6) as regard the age. Claiming that the age has been used in the past-in the absence of knowledge of exact time of disease onset-as a surrogate marker of disease duration and has been associated with more advanced fibrosis [20]. It also contradicts with Sumida et al., 2012 [21].

There was statistically significant difference on comparing group Ia with group Ib and group IIa with group IIb as regard the AST and the platelet count. This was in agreement with Sterling et al., 2006 [10] and Sumida et al., 2012 [21]. Platelet count is known to correlate with the amount of portal hypertension and advanced fibrosis [20].

There was no statistically significant difference when group Ia was compared with group Ib and comparing group IIa with group IIb as regard the ALT. This was in agreement with (Sumida et al., 2012) [21] who conducted this study upon Japanese nonalcoholic fatty liver disease population. This contradict with Sterling et al., 2006 [10] who found that there was statistically significant difference between mild (Ishak score 0 - 1), moderate (Ishak score 2 - 3) and severe fibrosis (Ishak score 4 - 6) as regard the ALT. The discrepancy was probably because patients included in Sterling et al., 2006 study had HIV/HCV co infection which may be a cofactor in elevation of ALT. To be noted, elevations in AST more than ALT have been associated with more advanced fibrosis [12] and are in part related to delayed clearance of AST relative to ALT or to mitochondrial injury associated with more advanced fibrosis.

When we compared group Ia with group Ib we found that there was a statistical significant difference as regard the total billirubin, direct bilirubin, serum creatinine and white blood count. Whereas there was no statistically significant difference to serum albumin, prothrombin time, international normalization ratio (INR), alpha fetoprotein, and haemoglobin. This was in agreement with Sterling et al., 2006 [10] as regard the total bilirubin and INR but was against their results as regards serum albumin. However Sterling et al., 2006 [10] didn't include the direct bilirubin, serum creatinine, the alpha fetoprotein, haemoglobin nor the white blood count in his statistical analysis. Our results were also contradicting with Sumida et al., 2012 [21] as regard the albumin.

On comparing group IIa and group IIb, we found that there was a statistical significant difference as regard the total bilirubin, the direct bilirubin, serum albumin international normalization ration, alpha fetoprotein and white blood count. This was in agreement with Sterling et al., 2006 [10] except for direct bilirubin, alpha fetoprotein and white blood count which were not included in their statistical analysis. There was no statistically significant difference as regard the serum creatinine and hemoglobin.

There was a statistically significant elevation of FIB-4 index with more liver fibrosison comparing group Ia and Ib as well as IIa and IIb. This was in agreement with Sumida et al., 2012 [21].

In the current study, On Analyzing correlation between FIB-4 index value and different parameters of the patients in the study, we found that there was a high statistically significant positive correlation as regard the AST, the results of Fibroscan in K. pascal, total \& direct bilirubin and there was a high statistically significant negative correlation as regard platelet count and serum albumin. Whereas there was no statistically significant correlation as regard the gender, ALT, serum creatinine and Alpha fetoprotein.

Possible explanations for these correlations are:

- No sex preponderance occurs with HCV infection. In the third National Health and Nutrition Examination Survey, neither sex nor racial-ethnic group was independently associated with HCV infection. Therefore sex is not independently associated with HCV related hepatic fibrosis [21].

- Prothrombin concentration represents synthetic function of the liver and it is affected with the progression of fibrosis.

- Elevations in AST more than ALT have been associated with more advanced fibrosis [12] and are in part related to delayed clearance of AST relative to ALT or to mitochondrial injury associated with more advanced fibrosis.

- Platelet count is known to correlate with the amount of portal hypertension and advanced fibrosis [20].

- Serum Albumin decreases with the increase of fibrosis and cirrhosis, the decrease in serum albumin correlates with the chronicity of liver disease.

From the statistical point of view, in this study, the FIB-4 index proved to be sensitive and specific in differentiation between patient with no or mild fibrosis (Metavir F0-F1) and patients with significant fibrosis or cir- 
rhosis $(\mathrm{F} 2-\mathrm{F} 3-\mathrm{F} 4)(\mathrm{AUC}=91.6)$ with the best cutoff value at 1.61 where sensitivity $69.5 \%$ was and specificity was $100 \%$. The positive predictive value was $100 \%$ to detect patient with no or mild fibrosis. The FIB-4 index also proved to be sensitive and specific in differentiation between patient with no or significant fibrosis (Metavir F0-F1-F2-F3) and patients with cirrhosis (Metavir F4) (AUC = 90.5) with the best cutoff value at 1.88 where sensitivity $84.6 \%$ was and specificity was $88.2 \%$. The positive predictive value was $88.2 \%$ and the negative predictive value was $100 \%$ to exclude patient with cirrhosis.

Using these cutoffs (1.61 - 1.88), 87\% of patients fell outside these ranges and could thus avoid biopsy with an overall accuracy of $70 \%$. The limitation of FIB-4 index is regarded to the patients who fell in the range between 1.61 and 1.88, thus require liver biopsy or Fibroscan for more accurate classification.

Sterling et al. (2006) [10] who proposed the use of FIB-4 index in patients with HIV/HCV coinfection, found that FIB-4 index can differentiate between Ishak stage 0 - 3 and $4-6$. At a cutoff of $<1.45$, the negative predictive value to exclude advanced fibrosis (stage 4 - 6) was $90 \%$ with a sensitivity of $70 \%$. A cutoff of $>3.25$ had a positive predictive value of $65 \%$ and a specificity of $97 \%$.

Vallet-Pichard et al. (2007) [11] evaluated the use of FIB-4 index in 847 patients with HCV monoinfection, found that FIB-4 index $<1.45$ had a negative predictive value of $94.7 \%$ to exclude severe fibrosis with a sensitivity of $74.3 \%$. An FIB-4 index higher than 3.25 had a positive predictive value to confirm the existence of a significant fibrosis (F3-F4) of $82.1 \%$ with a specificity of $98.2 \%$.

Sumida et al. (2012) [21] evaluated the use of FIB-4 in 576 patients with nonalcoholic fatty liver disease, found that FIB-4 index $<1.45$ had a negative predictive value of $98 \%$ to exclude severe fibrosis with a sensitivity of $90 \%$ and specificity of $64 \%$. An FIB-4 index higher than 3.25 had a positive predictive value to confirm the existence of a significant fibrosis (F3-F4) of 53\% with a specificity of $95 \%$.

\section{Conclusion}

The FIB-4 index is a new noninvasive test for the assessment of liver fibrosis. A score of 1.61 enables the correct identification of patients who have significant fibrosis and 1.88 enables the correct identification of patients who have cirrhosis.

\section{References}

[1] Pybus, O.G., Drummond, A.J., Nakano, T., et al. (2003) The Epidemiology and Iatrogenic Transmission of Hepatitis C Virus in Egypt: A Bayesian Coalescent Approach. Molecular Biology and Evolution, 20, 381-387. http://dx.doi.org/10.1093/molbev/msg043

[2] Saadeh, S., Cammell, G., Carey, W.D., et al. (2001) The Role of Liver Biopsy in Chronic Hepatitis C. Hepatology, 33, 196-200.

[3] Ishak, K., Baptista, A., Bianchi, L., et al. (1995) Histological Grading and Staging of Chronic Hepatitis. Journal of Hepatology, 22, 696-699. http://dx.doi.org/10.1016/0168-8278(95)80226-6

[4] Bedossa, P. and Poynard, T. (1996) An Algorithm for the Grading of Activity in Chronic Hepatitis C. The METAVIR Cooperative Study Group. Hepatology, 24, 289-293.

[5] Regev, A., Berho, M., Jeffers, L.J., et al. (2002) Sampling Error and Intraobserver Variation in Liver Biopsy in Patients with Chronic HCV Infection. The American Journal of Gastroenterology, 97, 2614-2618. http://dx.doi.org/10.1111/j.1572-0241.2002.06038.x

[6] Ziol, M., Handra-Luca, A., Kettaneh, A., et al. (2005) Noninvasive Assessment of Liver Fibrosis by Measurement of Stiffness in Patients with Chronic Hepatitis C. Hepatology, 41, 48-54. http://dx.doi.org/10.1002/hep.20506

[7] Sandrin, L., Oudry, J., Bastard, C., et al. (2011) Non-Invasive Assessment of Liver Fibrosis by Vibration-Controlled Transient Elastography (Fibroscan $^{\circledR}$ ). In: Takahashi, H., Ed., Liver Biopsy, InTech, Chapter 19. http://dx.doi.org/10.5772/20729

[8] Arena, U., Vizzutti, F., Corti, G., et al. (2008) Acute Viral Hepatitis Increases Liver Stiffness Values Measured by Transient Elastography. Hepatology, 47, 380-384. http://dx.doi.org/10.1002/hep.22007

[9] Torriani, F.J., Rodriguez-Torres, M., Rockstroh, J.K., Lissen, et al. (2004) Peginterferon Alfa-2a plus Ribavirin for Chronic Hepatitis C Virus Infection in HIV-Infected Patients. The New England Journal of Medicine, 351, 438-450. http://dx.doi.org/10.1056/NEJMoa040842

[10] Sterling, R.K., Lissen, E., Clumeck, N., et al. (2006) Development of a Simple Noninvasive Index to Predict Significant Fibrosis in Patients with HIV/HCV Coinfection. Hepatology, 43, 1317-1325. http://dx.doi.org/10.1002/hep.21178 
[11] Vallet-Pichard, A., Mallet, V., Nalpas, B., Verkarre, V., Nalpas, A., Dhalluin-Venier, V., et al. (2007) FIB-4: An Inexpensive and Accurate Marker of Fibrosis in HCV Infection. Comparison with Liver Biopsy and Fibrotest. Hepatology, 46, 32-36. http://dx.doi.org/10.1002/hep.21669

[12] Pohl, A., Behling, C., Oliver, D., Kilani, M., Monson, P. and Hassanein, T. (2001) Serum Aminotransferase Levels and Platelet Counts as Predictors of Degree of Fibrosis in Chronic Hepatitis C Virus Infection. The American Journal of Gastroenterology, 96, 3142-3146. http://dx.doi.org/10.1111/j.1572-0241.2001.05268.x

[13] Kelleher, T.B., Mehta, S.H., Bhaskar, R., Sulkowski, M., Astemborski, J., Thomas, D.L., et al. (2005) Prediction of Hepatic Fibrosis in HIV/HCV Co-Infected Patients Using Serum Fibrosis Markers: The SHASTA Index. Journal of Hepatology, 43, 78-84. http://dx.doi.org/10.1016/j.jhep.2005.02.025

[14] Hatzakis, A., Wait, S., Bruix, J., Buti, M., Carballo, M., Cavaleri, M., et al. (2011) The State of Hepatitis B and C in Europe: Report from the Hepatitis B and C Summit Conference. Journal of Viral Hepatitis, 18, 1-16.

[15] Lavanchy, D. (2009) The Global Burden of Hepatitis C. Liver International, 29, 74-81. http://dx.doi.org/10.1111/j.1478-3231.2008.01934.x

[16] Shepard, C.W., Finelli, L. and Alter, M.J. (2005) Global Epidemiology of Hepatitis C Virus Infection. The Lancet Infectious Diseases, 5, 558-567. http://dx.doi.org/10.1016/S1473-3099(05)70216-4

[17] Kamal, S.M. and Nasser, I.A. (2008) Hepatitis C Genotype 4. What We Know and What We Don’t Yet Know. Hepatology, 47, 1371-1383.

[18] Massard, J., Ratziu, V., Thabut, D., Moussalli, J., Lebray, P., Benhamou, Y. and Poynard, T. (2006) Natural History and Predictors of Disease Severity in Chronic Hepatits C. Journal of Hepatology, 44, S19-S24. http://dx.doi.org/10.1016/j.jhep.2005.11.009

[19] Wait, C.T., Greeson, J.K., Fontana, R.J., Kalbfleisch, J.D., Marrero, J.A., Conjeevaram, H.S. and Lok, A.S. (2003) A Simple Non Invasive Index Can Predict both Significant Fibrosis and Cirrhosis with Chronic Hepatitis C. Hepatology, 38, 518-526. http://dx.doi.org/10.1053/jhep.2003.50346

[20] Sterling, R.K., Stravitz, R.T., Luketic, V.A., Sanyal, A.J., Contos, M.J., Mills, A.S. and Shiffman, M.L. (2004) A Comparison of the Spectrum of Chronic Hepatitis C Virus between Caucasians and African Americans. Clinical Gastroenterology and Hepatology, 2, 469-473. http://dx.doi.org/10.1016/S1542-3565(04)00164-8

[21] Sumida, Y., Yoneda, M., Hyogo, H., Itoh, Y., Ono, M., Fujii, H., et al. (2012) Validation of the FIB4 Index in a Japanese Nonalcoholic Fatty Liver Disease Population. BMC Gastroenterology, 12, 2. 
Scientific Research Publishing (SCIRP) is one of the largest Open Access journal publishers. It is currently publishing more than 200 open access, online, peer-reviewed journals covering a wide range of academic disciplines. SCIRP serves the worldwide academic communities and contributes to the progress and application of science with its publication.

Other selected journals from SCIRP are listed as below. Submit your manuscript to us via either submit@scirp.org or Online Submission Portal.
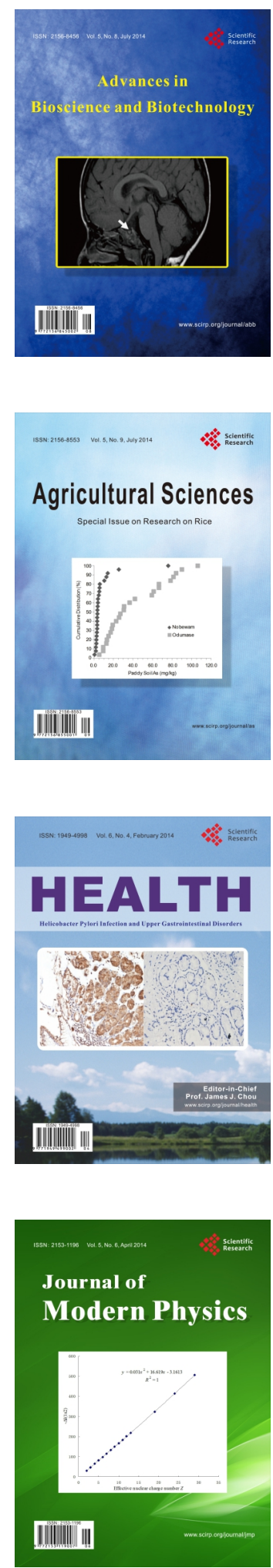
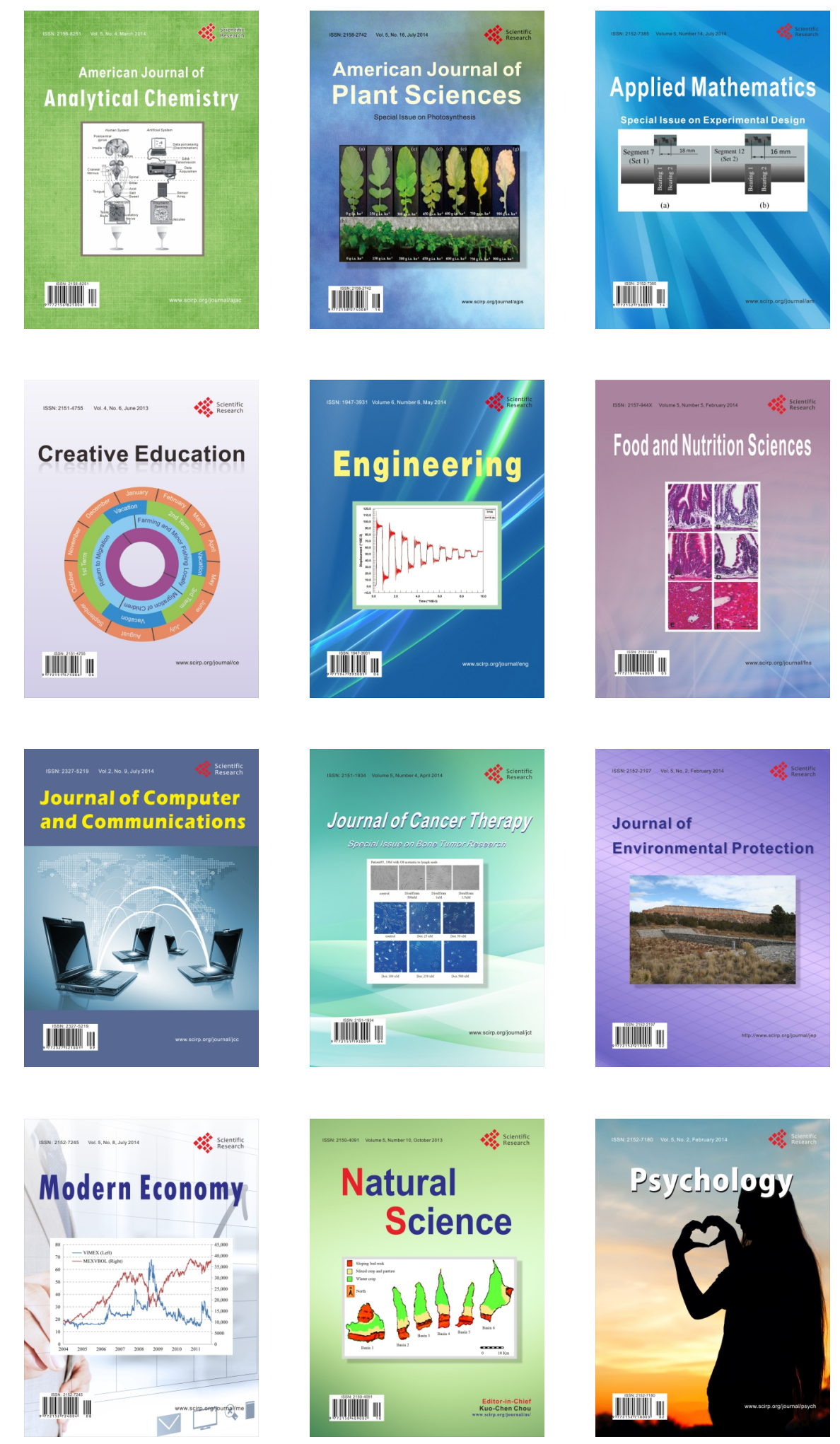